\title{
Synthesis and Liquid Chromatographic Determination of Optical Purity of Naphthyl Propionate Liquid Crystals ${ }^{\dagger}$
}

\author{
Seng Kue Lee, Myung Soo Shin, Jong Gun Lee, Kyung-Tae Kang, and Yong Bae Kim \\ Department of Chemistrv and Chemistry Institute for Functional Materials, Pusan National Cniversit, Pusan 609-735, Korea \\ -Liquid Crystal Research Center and Department of Chemisty, Konkuk Chiversitw. Seoul 143-710, Korea \\ Received April 6. 2004
}

Key Words : Chiral stationary phase. Chiral separation. Liquid chromatographỵ. Ferroelectric liquid crysstal. (S)-Naproxen

Most ferro- and antiferroelectric liquid crystals are chiral and their mesomorphic phase structures and electro-optical properties are largely dependent on the optical purity. ${ }^{1,2}$ Thus, for the chiral liquid crystals the occurrence of chemical or thernal racentization has to be checked throughout the synthetic sequence and the investigation of the mesomorphic and electro-optical properties.

However, most research papers on the chiral liquid crystals did not mention the enantioneric excess of the materials. They were just assumed to be the same as those of the starting materials or of various intermediates without any investigation. It is very important to establish convenient and accurate means of determining the enantiomeric conposition in liquid crystal chemistry. Among various techniques. liquid chromatographic separation of enantiomers on the chiral stationary phases (CSPs) night be the choice because this technique is known to be the most simple and convenient means of determining the enantiomeric composition. ${ }^{3}$ In this study. we show that a conmercial HPLC chiral column. $(S, S)$-Whelk-Ol. can resolve various types of
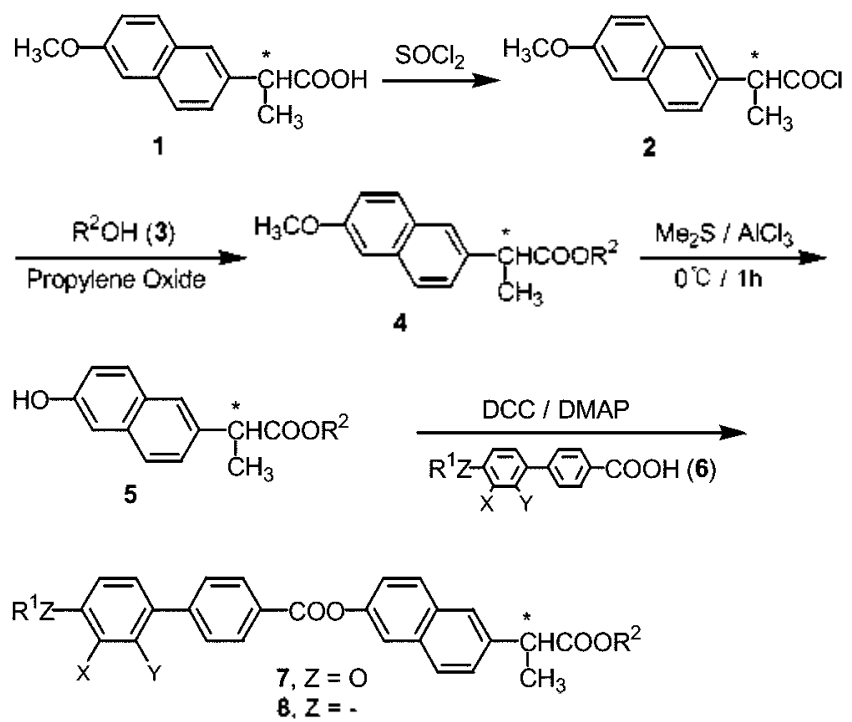

Scheme 1

"This paper is dedicated to Professor Yone Hae Kim for his distinguished achievements in chemistry.

"Corresponding Author. e-mail: krthang octusan.ac.kr naphthyl propionate liquid crystals.

Naphthyl propionate ferroelectric liquid crystals with an ester linkage between naphthalene and biphenyl rings were prepared from $(S)$-naproxen 1 as depicted in Scheme . $^{4}$

Since the hydrogen atom on the chiral carbon in the naproxen derivatives 2. 4. 5,7 and 8 is acidic enough due to the presence of $\alpha$-naphthalene ring and either carbosyl or carboalkoxy group. racemization wia enolization in the compounds can take place routinely under both acidic and basic reaction conditions. Thus one has to confirm the integrity of chiral center in the intermediate and final products by the HPLC analy sis using chiral colunu.

The integrity of chiral center in the reaction products 4 prepared from (S)-naproxen chloride (2) with alcohol in the presence of propylene oxide." was confirmed by the HPLC. Racemic naproxen was obtained from (S)-naproxen (1) by deprotonating the acidic hydrogen with litlium diisopropylamide (LDA) followed by acidification and base-catalyzed racemization in aprotic polar solvents. Thus obtained racemic naproxen was converted to the racenic $n$-butyl ester ta $\left(\mathrm{R}^{2}=\mathrm{C}_{4} \mathrm{H}_{0}\right)$ according to Scheme 1 . HPLC resolution of racemic ester $4 a$ on a chiral column showed a reasonable separation factor [Figure l(a)]. Figure $l(b)$ shows that ta prepared from (S)-naproxen was optically pure (enantiomeric excess: $>99 \%$ ee); racemization did not occur at all during the formation of ta from (S)-naproxen.

Demethylation of methosy group of the esters 4 by dimethylsulfide- $\mathrm{AlCl}_{3}$ produced naphthols 5 in good yields with no actual racemization [Figure l(c)]. Demethylation of ta with boron tribronide gave $\mathbf{5 a}$ in much lower yield $(50.3 \%)$.

Esterifications of (S)-naproxen (1) with alcolnols were achieved by using DCC and DMAP as described in the literatures $^{7.31}$ racemizations occurred in some extent (Scheme 2). Contrary to the above results. the racemization was not almost observed in the esterification of $\mathbf{5}$ with $\mathbf{6}$ using DCC and DMAP to give optically pure 7 a ( $99 \%$ ee) [Figure $\mathrm{I}(\mathrm{d})$ ].

The specific rotation of compound $7 \mathrm{e}\left(\mathrm{R}^{1}=\mathrm{C}_{10} \mathrm{H}_{21}\right.$, $\mathrm{R}^{2}=\mathrm{C}_{4} \mathrm{H}_{9}, \mathrm{X}=\mathrm{Y}=\mathrm{H}$ ) prepared by us showed $[\alpha]_{\mathrm{D}}^{175}=+17.06$, which is much higher than the reported value of $[\alpha]_{01}^{23}=$ $+4.93{ }^{10}$ This proves that the compounds prepared in this study are of better optical purity for investigating plysical 

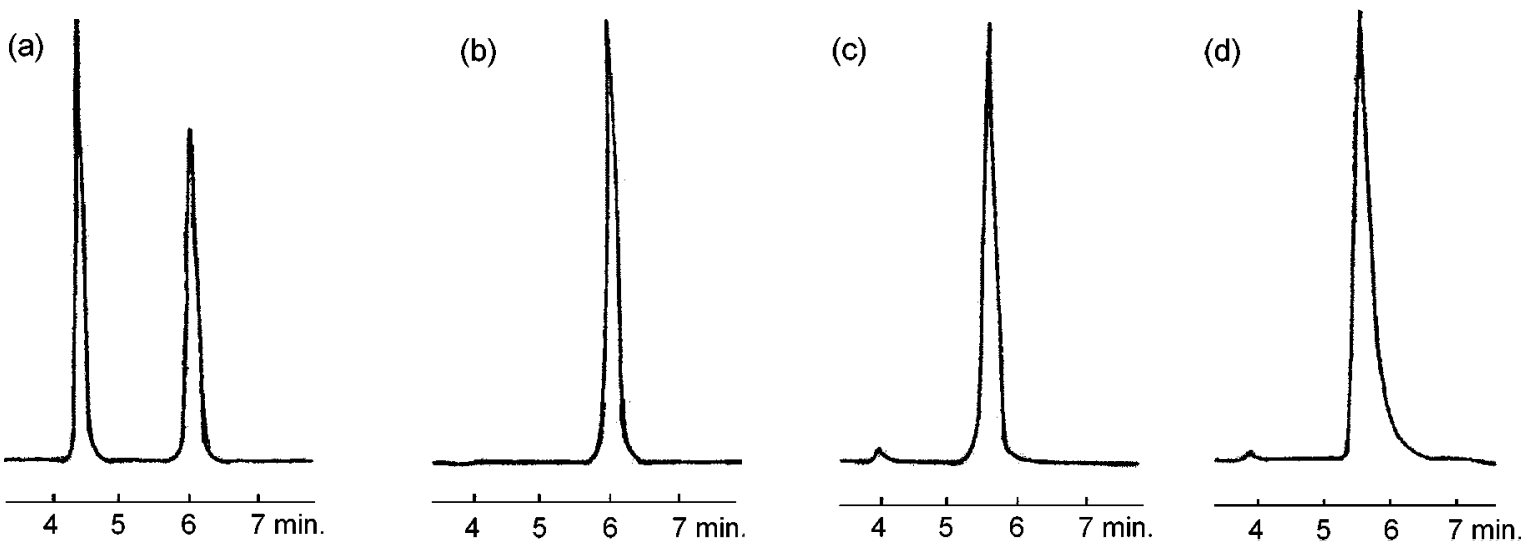

Figure 1. Chromatograms for resolving ester ta of (a) racemic $4 a_{\text {, (b) }} 4 \mathbf{a}_{\text {, }}$ (c) $5 \mathbf{a}_{\text {, and }}$ (d) 7 a prepared from (S)-naproxen through Scheme 1

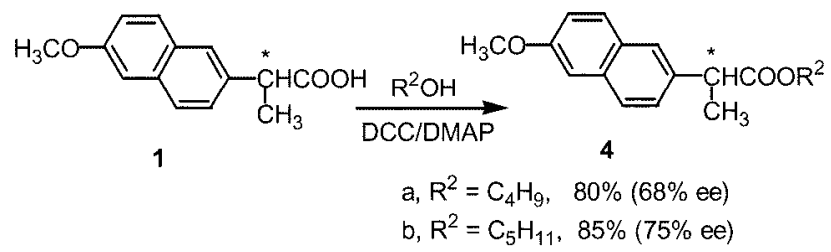

Scheme 2

Table 1. Liquid chromatographic resolution of naphthyl propionate liquid crystals 7 and 8 on $(S, S)$-Whelk-Ol chiral columnin"

\begin{tabular}{|c|c|c|c|c|c|c|c|}
\hline $\begin{array}{l}\text { Liquid } \\
\text { Crystal }\end{array}$ & $\mathrm{R}^{1}$ & $\mathrm{R}^{2}$ & $X$ & Y & $\mathrm{k}_{1}^{b}$ & $\mathrm{k}_{2}^{b}$ & $\alpha$ \\
\hline $7 a$ & $\mathrm{C}_{8} \mathrm{H}_{1} ;$ & $\mathrm{C}_{4} \mathrm{H}_{0}$ & $\mathrm{H}$ & $\mathrm{H}$ & 3.17 & 4.91 & 1.55 \\
\hline $7 b$ & $\mathrm{C}_{8} \mathrm{H}_{1}:$ & $\mathrm{C}_{5} \mathrm{H}_{1 \mathrm{l}}$ & $\mathrm{H}$ & $\mathrm{H}$ & 3.12 & 4.79 & 1.54 \\
\hline $7 \mathrm{c}$ & $\mathrm{C}_{8} \mathrm{H}_{1}=$ & $\mathrm{C}_{6} \mathrm{H}_{13}$ & $\mathrm{H}$ & $\mathrm{H}$ & 3.03 & 4.19 & 1.38 \\
\hline $7 d$ & $\mathrm{C}_{0} \mathrm{H}_{10}$ & $\mathrm{C}_{4} \mathrm{H}_{0}$ & $\mathrm{H}$ & $\mathrm{H}$ & 4.27 & 5.33 & 1.06 \\
\hline $7 e$ & $\mathrm{C}_{10} \mathrm{H}_{21}$ & $\mathrm{C}_{4} \mathrm{H}_{0}$ & $\mathrm{H}$ & $\mathrm{H}$ & 3.45 & 4.84 & 1.40 \\
\hline $7 \mathbf{f}$ & $\mathrm{C}_{\mathrm{S}} \mathrm{H}_{1}=$ & $\mathrm{C}_{4} \mathrm{H}_{\vartheta}$ & $\mathrm{F}$ & $\mathrm{H}$ & 4.14 & 5.79 & 1.40 \\
\hline $7 \mathrm{~g}$ & $\mathrm{C}_{\mathrm{S}} \mathrm{H}_{1}=$ & $\mathrm{C}_{\leq} \mathrm{H}_{[1]}$ & $\mathrm{F}$ & $\mathrm{H}$ & 3.21 & 5.20 & 1.62 \\
\hline $7 \mathrm{~h}$ & $\mathrm{C}_{\mathrm{S}} \mathrm{H}_{1}=$ & $\mathrm{C}_{4} \mathrm{H}_{4}$ & $\mathrm{~F}$ & F & 3.46 & 4.71 & 1.36 \\
\hline $7 i$ & $\mathrm{C}_{\mathrm{S}} \mathrm{H}_{1}=$ & $\mathrm{C}_{4} \mathrm{H}_{[1]}$ & $\mathrm{F}$ & F & 3.02 & 3.97 & 1.31 \\
\hline $8 a$ & $\mathrm{C}_{\mathrm{S}} \mathrm{H}_{1}=$ & $\mathrm{C}_{4} \mathrm{H}_{4}$ & $\mathrm{H}$ & $\mathrm{H}$ & 2.29 & 3.37 & 1.47 \\
\hline $8 b$ & $\mathrm{C}_{\mathrm{S}} \mathrm{H}_{1}=$ & $\mathrm{C}_{\leq} \mathrm{H}_{[1]}$ & $\mathrm{H}$ & $\mathrm{H}$ & 2.26 & 3.15 & 1.39 \\
\hline $8 c$ & $\mathrm{C}_{\mathrm{S}} \mathrm{H}_{1}=$ & $\mathrm{C}_{n} \mathrm{H}_{13}$ & $\mathrm{H}$ & $\mathrm{H}$ & 2.00 & 2.95 & 1.48 \\
\hline
\end{tabular}

"See the Experimental part for the chromatographic condition. ${ }^{b}$ Retention factor of the first and second eluted enantiomers. "Separation factor.

properties. The results of chromatographic resolution are summarized in Table 1.

Naphthyl propionates having an ethereal linkage 10 were prepared from Mitsunobu reaction of naphthols 5 with 4-(4alkoxyphenyl)benzyl alcohols 9 (Scheme 3). Although the yields were low. optically pure products 10 were obtained. Their optical purites were estimated in the same manner using the same HPLC chiral column. (S,S)-Whelk-O1 (Table 2). The yields of the ether $\mathbf{1 0}$ were improved $(>72 \%)$ in the reactions of naphthol 5 with 4-(4-alkoxyphenyl)benzyl chloride in the presence of potassium carbonate. however. complete racemizations were occurred.

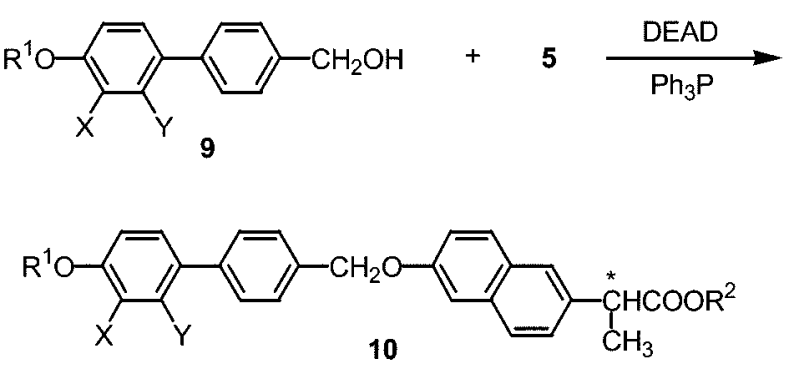

Scheme 3

Thus obtained naphthyl propionate ferroelectric liquid crystals having an oxymethylene linking group 10 exhibited an enantiotropic mesogenic phases with the phase sequence: $\mathrm{Cry}-\mathrm{Sc}^{*}-\mathrm{S}_{\mathrm{A}}-\mathrm{Iso}$. But the compound 10a with short alkyl chain as teminal group does not show the chiral smectic $C$ phase. The naphthyl propionates with a fluorinated biphenyl group 10h-10l have lower transition temperature than the nonfluorinated compounds 10a-10g. Table 3 summarizes the mesomorphic transition temperatures and enthalpies for the

Table 2. Liquid chromatographic resolution of naphthyl propionate liquid erystals 10 on $(S, S)$-Whelk-Ol chiral column"

\begin{tabular}{|c|c|c|c|c|c|c|c|}
\hline $\begin{array}{l}\text { Liquid } \\
\text { Crystal }\end{array}$ & $\mathrm{R}^{\mathrm{l}}$ & $\mathrm{R}^{2}$ & $X$ & $\mathrm{Y}$ & $\mathrm{k}_{\mathrm{l}}{ }^{t}$ & $\mathrm{k}_{2}{ }^{4}$ & $\alpha$ \\
\hline $10 a$ & $\mathrm{C}_{8} \mathrm{H}_{1}$ ? & $\mathrm{C}_{4} \mathrm{H}_{4}$ & $\mathrm{H}$ & $\mathrm{H}$ & 1.76 & 2.81 & 1.60 \\
\hline $10 \mathrm{~b}$ & $\mathrm{C}_{8} \mathrm{H}_{1}=$ & $\mathrm{C}_{5} \mathrm{H}_{11}$ & $\mathrm{H}$ & $\mathrm{H}$ & 1.60 & 2.61 & 1.63 \\
\hline $10 c$ & $\mathrm{C}_{8} \mathrm{H}_{1}=$ & $\mathrm{C}_{6} \mathrm{H}_{13}$ & $\mathrm{H}$ & $\mathrm{H}$ & 1.40 & 2.31 & 1.65 \\
\hline $10 \mathrm{~d}$ & $\mathrm{C}_{8} \mathrm{H}_{1}=$ & $\mathrm{C}^{\mathrm{C}}: \mathrm{H}_{15}$ & $\mathrm{H}$ & $\mathrm{H}$ & 1.28 & 2.15 & 1.68 \\
\hline $10 \mathrm{e}$ & $\mathrm{C}_{8} \mathrm{H}_{19}$ & $\mathrm{C}_{4} \mathrm{H}_{4}$ & $\mathrm{H}$ & $\mathrm{H}$ & 1.68 & 2.69 & 1.60 \\
\hline $10 f$ & $\mathrm{Co}_{19}$ & $\mathrm{C}_{3} \mathrm{H}_{11}$ & $\mathrm{H}$ & $\mathrm{H}$ & 1.46 & 2.37 & 1.62 \\
\hline $10 \mathrm{~g}$ & $\mathrm{C}_{9} \mathrm{H}_{19}$ & $\mathrm{C}_{5} \mathrm{H}_{13}$ & $\mathrm{H}$ & $\mathrm{H}$ & 1.35 & 2.26 & 1.67 \\
\hline $10 \mathrm{~h}$ & $\mathrm{C}_{8} \mathrm{H}_{1}$ ? & $\mathrm{C}_{3} \mathrm{H}_{1 \mathrm{l}}$ & $\mathrm{F}$ & $\mathrm{H}$ & 1.77 & 2.86 & 1.62 \\
\hline $10 \mathrm{i}$ & $\mathrm{C}_{y} \mathrm{H}_{19}$ & $\mathrm{C}_{3} \mathrm{H}_{1 \mathrm{l}}$ & $\mathrm{F}$ & $\mathrm{H}$ & 1.70 & 2.77 & 1.63 \\
\hline $10 \mathrm{j}$ & $\mathrm{C}_{10} \mathrm{H}_{21}$ & $\mathrm{C}_{5} \mathrm{H}_{1 \mathrm{l}}$ & $\mathrm{F}$ & $\mathrm{H}$ & 1.68 & 2.72 & 1.62 \\
\hline $10 \mathrm{k}$ & $\mathrm{C}_{10} \mathrm{H}_{21}$ & $\mathrm{C}_{4} \mathrm{H}_{4}$ & $\mathrm{~F}$ & $\mathrm{H}$ & 1.85 & 2.93 & 1.58 \\
\hline 101 & $\mathrm{C}_{10} \mathrm{H}_{21}$ & $\mathrm{C}_{4} \mathrm{H}_{13}$ & $\mathrm{~F}$ & $\mathrm{H}$ & 1.49 & 2.50 & 1.68 \\
\hline
\end{tabular}

"See the Experimental part for the chromatographic condition. "Retention factor of the first and second el uted enantiomers. "Separation factor. 
Table 3. Transition temperatures $\left({ }^{\circ} \mathrm{C}\right)$ and enthalpies $\left(\Delta \mathrm{H} / \mathrm{kJmol}^{-1}\right)$ (in italics) of naphthy 1 propionates $\mathbf{1 0}$ on cooling

\begin{tabular}{|c|c|c|c|c|c|c|c|c|}
\hline $\begin{array}{l}\text { Liquid } \\
\text { Crystal }\end{array}$ & I & & $S_{A}$ & & $\mathrm{~S}_{1}{ }^{*}$ & & $\mathrm{Cr}_{\mathrm{I}}$ & $\mathrm{mp}$ \\
\hline \multirow[t]{2}{*}{$10 \mathrm{a}$} & . & 116.0 & & & - & 82.2 & & 107.5 \\
\hline & & 4.71 & & & & 25.2 & & \\
\hline \multirow[t]{2}{*}{$10 \mathrm{~b}$} & . & 116.9 & & 106.2 & . & 66.8 & & 100.9 \\
\hline & & 6.33 & & 0.11 & & 25.2 & & \\
\hline \multirow[t]{2}{*}{$10 \mathrm{c}$} & . & 113.2 & 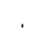 & 97.9 & . & 75.5 & & 98.7 \\
\hline & & 6.76 & & 0.04 & & 23.9 & & \\
\hline \multirow[t]{2}{*}{$10 \mathrm{~d}$} & . & 115.7 & . & 96.3 & & 77.1 & & 96.7 \\
\hline & & 6.81 & & 1.32 & & 19.8 & & \\
\hline \multirow[t]{2}{*}{$10 \mathrm{e}$} & . & 118.1 & . & 113.3 & & 85.1 & & 112.7 \\
\hline & & 6.15 & & 0.39 & & 20.3 & & \\
\hline \multirow[t]{2}{*}{$10 \mathrm{f}$} & . & 112.8 & . & 1053 & . & 79.2 & . & 96.5 \\
\hline & & 5.86 & & 0.26 & & 20.7 & & \\
\hline \multirow[t]{2}{*}{$10 \mathrm{~g}$} & . & 111.2 & . & 101.7 & . & 74.2 & & 99.2 \\
\hline & & 6.25 & & 0.18 & & 19.2 & & \\
\hline \multirow[t]{2}{*}{$10 \mathrm{~h}$} & . & 88.4 & . & 73.2 & . & 41.3 & & 82.7 \\
\hline & & 4.70 & & 0.11 & & 21.7 & & \\
\hline \multirow[t]{2}{*}{$10 i$} & . & 87.0 & . & 74.9 & & 43.4 & & 75.1 \\
\hline & & 4.75 & & 0.11 & & 23.4 & & \\
\hline \multirow[t]{2}{*}{$10 \mathrm{j}$} & . & 88.7 & . & 78.1 & & 47.5 & . & 75.3 \\
\hline & & 5.19 & & 0.10 & & 26.4 & & \\
\hline \multirow[t]{2}{*}{$10 k$} & . & 91.3 & . & 82.2 & . & 52.3 & & 71.9 \\
\hline & & 4.96 & & 0.11 & & 26.7 & & \\
\hline \multirow[t]{2}{*}{101} & . & 88.6 & 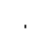 & 75.9 & . & 47.2 & & 66.2 \\
\hline & & 5.03 & & 0.12 & & 28.2 & & \\
\hline
\end{tabular}

$\mathrm{Cr}_{\mathrm{r}}=$ crystalline phase: $\mathrm{S}_{\mathrm{r}}^{*}=$ chiral smectic $\mathrm{C}$ phase: $\mathrm{S}_{\mathrm{A}}=$ smectic $\mathrm{A}$ phase: I = isotropic liquid phase.

alkyl (S)-2-\{6-[4-(4'-alky loxybipheny l)benzy loxy $]-2$ naphthyl\}propionates 10. Exchanging of central linkage group from ester (compounds 7 and 8 ) to ether (compound 10) in the naphthyl propionate liquid crystals, the temperature range of the $S_{\mathrm{A}}$ phase became narrower and the $\mathrm{N}^{*}$ phase was not appeared.

In summary. various types of optically pure naphthyl propionate liquid crystals were prepared from (S)-naproxen in a four step reaction sequence. We found that a commercial HPLC chiral column. (S,S)-Whelk-O1. successfully resolves the (S)-naproxen-based liquid crystals. This teclunique is very simple and effective in monitoring the enantiomeric excess of the intermediate and final products without any structural modification at all

\section{Experimental Section}

${ }^{l}$ H-NMR spectra were recorded on Varian Gemini-200 $(200 \mathrm{MHz})$ and Varian Inova $(500 \mathrm{MHz})$ spectrometer using chloroform as an internal standard. The latter instrument was also used for recording ${ }^{13} \mathrm{C}-\mathrm{NMR}$ spectra in $\mathrm{CDCl}_{\hat{3}}$ (solvent and internal reference) and ${ }^{l,} \mathrm{~F}$-NMR spectra in $\mathrm{CDCl}_{3}$ (trifluoroacetic acid as an internal reference). Elemental analyses were performed at the National Center for InterUniversity Research Facilities. Seoul National University.
High mass spectra were taken on a Jeol JMS-700 spectrometer. Phase transition temperature and phase appearance of final products were measured by using polarizing microscope (Olympus $\mathrm{BH}-2$ ) with a hot stage and a controller (Mettler FP 90). Transition temperature and enthalpy were determined by differential scanning calorimetry (DSC) using a Perkin Elmer DSC 7 calorimeter. HPLC analyses were performed with an instrument consisted of Waters model 510 pump, a Rheodyne model 7125 injector with a $20 \mu \mathrm{L}$ sample loop, a Youngin model 710 absorbance detector with a $254 \mathrm{~nm}$ UV filter and a Youngin D520B computing integrator. A mixed solvent of isopropyl alcohol and hexanes $(80: 20 . \mathrm{v} / \mathrm{v})$ was used as a mobile phase with a flow rate of $2.0 \mathrm{~mL} / \mathrm{min}$ at room temperature. The column void volume was determined by injecting 1,3.5-tri-ten-butylbenzene. a presumed unretained solute. ${ }^{12}$ The chiral column (S.S) Whelk-Ol, used in this study is conmercially available from Regis Technologies, Inc. (Morton Grove, Illinois. USA). Optical rotations were determinated as a solution in chloroform using a JASCO DIP- 1000 digital polarimeter with a path length of $\mathrm{l} \mathrm{dm}$. Concentrations are quoted in $\mathrm{g} / 100 \mathrm{~mL}$. (S)-Naproxen (1. > 98\% ee) was purchased from Aldrich Chemical $\mathrm{Co}_{\text {., }}$ and used without further purification.

Synthesis of Alkyl (S)-2-\{6[4-(4'-Alkyloxyphenyl)benzyloxy]-2-naphthyl\}propionate 10. A typical procedure for the synthesis of $10 \mathrm{a}$ is following. To a THF $(5 \mathrm{~mL})$ solution of 4-(4'-octyloxyphenyl)benzyl alcohol 7a $(0.20$ g. 0.59 mumol) was added triphenyl phosphine $(0.17 \mathrm{~g} .0 .65 \mathrm{mmol})$ and diethyl azodicarboxylate (DEAD. $0.11 \mathrm{~g} .0 .64 \mathrm{mmol}$ ) and stirred at room temperature for $\mathrm{l} \mathrm{h}$. To the mixture butyl (5)-2-(6-lydroxy-2-naphthyl)propionate 5a $(0.17$ g. 0.62 mmol) in THF ( $2 \mathrm{~mL}$ ) was added and stirred for 24 lurs at room temperature. The precipitated triphenylphosphine oxide and diethyl hydrazinedicarboxylate were removed by filtration. The filtrate was concentrated in vacto and the residue was chromatographed on silica gel $\left(\mathrm{CH}_{2} \mathrm{Cl}_{2}\right)$ to give $0.09 \mathrm{lg}(25 \%)$ of $10 \mathrm{a}$. Compounds 10b-10l were similarly prepared.

10a: ${ }^{~} \mathrm{H}-\mathrm{NMR} \delta 0.81-1.79$ (m. 25H). 3.84 (q. 1H. $J=7.0$ Hz). $3.94-4.10(\mathrm{~m} .4 \mathrm{H}) .5 .18$ (s. $2 \mathrm{H}) .6 .95-7.74(\mathrm{~m} .14 \mathrm{H})$ : ${ }^{13}$ C.-NMR $\delta 13.6,14.1 .18 .5,19.0 .22 .6 .26 .1,29.2,29.3$. $29.4,30.6,31.8,45.5,64.6,68.1,69.9,107.0,114.8,119.3$. 125.9, 126.3, 126.9. 127.1. 128.0, 128.1, 129.1, 129.3. $133.1,133.6,135.1 .136 .0,140.7 .158 .7,158.8 .174 .7:[\alpha]_{D}^{122}$ $=-5.5$ (C 0.29): Anal. Calc. for $\mathrm{C}_{38} \mathrm{H}_{46} \mathrm{O}_{4}: \mathrm{C} 80.53$. H 8.18 found $\mathrm{C} 80.38$. H 8.22 .

10b: ${ }^{~} \mathrm{H}-\mathrm{NMR} \delta 0.78-1.79$ (m. 27H). 3.85 (q. $1 \mathrm{H} . J=7.0$ Hz). 3.96-4.10 (m. 4H). 5.19 (s. 2H). 6.95-7.75 (m. 14H): ${ }^{13}$ C-NMR $\delta 13.8,14.1,18.5,22.2 .22 .6 .26 .0 .27 .9,28.2$. 29.2. 29.3. 29.4, 31.8, 45.5, 64.9. 68.1. 69.9. 107.0. 114.8. $119.3,125.9,126.3,126.9 .127 .1,128.0 .128 .1 .129 .1$. $129.3,133.1,133.6,135.1,136.0,140.7,156.7,158.8$. 174.7: $[\alpha]_{5 j}^{33}=-12.8$ (C 0.24): Anal. Calc. for $\mathrm{C}_{39} \mathrm{H}_{46} \mathrm{O}_{4}: \mathrm{C}$ 80.65. H 8.33: found C 80.57. H 8.33 .

10c: ${ }^{1} \mathrm{H}-\mathrm{NMR} \delta 0.79-1.79$ (m, 29H). 3.81 (q. 1H. $J=7.0$ Hz). $3.94-4.07$ (m. $4 \mathrm{H}), 5.18$ (s. 2H). 6.92-7.32 (m. 14H): ${ }^{13} \mathrm{C}-\mathrm{NMR} \delta 13.9,14.1 .18 .5 .22 .4 .22 .6 .25 .4 .26 .1,28.5$. 
$29.2,29.3,29.4,31.3,31.8,45.5,64.9,68.1,69.9,107.0$. $114.8,119.3 .125 .9,126.3,126.9 .127 .1,128.0,128.1$. 129.1, 129.3. 133.1, 133.6, 135.1. 136.0, 140.7, 156.8. 158.8, 174.7: $[\alpha]_{D}^{2-1}=-13.5(\mathrm{C} 0.10)$ : Anal. Calc. for $\mathrm{C}_{413} \mathrm{H}_{5(\mathrm{O}} \mathrm{O}_{4}: \mathrm{C} 80.77, \mathrm{H} 8.47$; found $\mathrm{C} 80.59, \mathrm{H} 8.57$.

10d: ${ }^{1} \mathrm{H}-\mathrm{NMR} \delta 0.82-1.79(\mathrm{~m} .3 \mathrm{lH}$ ). 3.84 (q. IH. $J=7.0$ Hz). 3.98-4.07 (nt, +H). 5.19 (s. 2H). 6.93-7.73 (n, l4H): ${ }^{13} \mathrm{C}-\mathrm{NMR} \delta 14.0,14.1,18.5,22.5,22.6 .25 .7 .26 .1 .28 .5$. $28.8,29.2,29.3,29.7,31.6,31.8,45.6,64.9,681,69.9$. $107.1,114.8 .119 .3,125.9,126.3 .126 .9,127.1,128.0$. 128.1, 129.1. 129.3, 133.1, 133.6. 135.1, 136.0, 140.7. 156.8, 158.8. 174.7; $[\alpha]_{D}^{20}=-13.2(\mathrm{C}$ 0.11): Anal. Calc. for $\mathrm{C}_{41} \mathrm{H}_{52} \mathrm{O}_{4}: \mathrm{C} 80.88, \mathrm{H} 8.61$ : found $\mathrm{C} 80.07, \mathrm{H} 8.73$.

10e: ${ }^{1} \mathrm{H}-\mathrm{NMR} \delta 0.82-1.83(\mathrm{~m}, 27 \mathrm{H}) .3 .84$ (q. $\mathrm{lH} . \quad J=7.0$ Hz). 3.96-4.07 (n, $4 \mathrm{H}) .5 .18$ (s. 2H). 6.94-7.74 (n, l4H): ${ }^{13} \mathrm{C}-\mathrm{NMR} \delta 13.6,14.1,18.5,19.0,22.7 .26 .1 .29 .2 .29 .3$. $29.4,29.5 .30 .6 .31 .9 .45 .5 .64 .6,68.1,69.9 .107 .0,114.9$. $119.3,125.9 .126 .3,126.9,127.1 .128 .0,128.1,129.1$. $129.3,133.1 .133 .6,135.1,136.0 .140 .7,156.8,158.8$. 174.7: $[\alpha]_{D}^{28}=-12.0\left(\mathrm{C} \mathrm{0.11)}\right.$; Anal. Calc. for $\mathrm{C}_{39} \mathrm{H}_{48} \mathrm{O}_{4}: \mathrm{C}$ $80.65, \mathrm{H} 8.33$ : found C $80.66, \mathrm{H} 8.36$.

10f: ${ }^{1} \mathrm{H}-\mathrm{NMR} \delta 0.78-1.82$ (n. $29 \mathrm{H}$ ), $3.82(\mathrm{q}, 1 \mathrm{H}, J=7.0$ Hz). 3.94-4.08 (m, $4 \mathrm{H}) .5 .19$ (s. 2H). 6.92-7.73 (n, l4H): ${ }^{13} \mathrm{C}-\mathrm{NMR} \delta 13.9,14.1,18.5,22.2,22.7 .26 .0 .27 .9 .28 .2$. $29.2,29.3,29.4,29.5,31.9,45.5,64.9,68.1,69.9,107.0$. $114.8,119.3 .125 .9,126.3,126.9 .127 .1,128.0,128.1$. $129.1,129.3 .133 .1,133.6,135.1 .136 .0,140.7,156.8$. 158.8, 174.7: $[\alpha]_{D}^{28}=-8.9$ (C 0.30$)$; Anal. Calc. for $\mathrm{C}_{410} \mathrm{H}_{5(\mathrm{O}} \mathrm{O}_{4}: \mathrm{C} 80.77, \mathrm{H} 8.47$; found $\mathrm{C} 80.59, \mathrm{H} 8.46$.

10g: ${ }^{1} \mathrm{H}-\mathrm{NMR} \delta 0.82-1.84(\mathrm{~m}, 31 \mathrm{H}), 3.84(\mathrm{q}, 1 \mathrm{H}, J=7.0$ Hz). 3.96-4.09 (nt, 4H). 5.18 (s. 2H). 6.94-7.75 (n, l4H): ${ }^{13} \mathrm{C}-\mathrm{NMR} \delta 13.9,14.1,18.5,22.5,22.7 .25 .4 .26 .1 .28 .5$. $29.3,29.3,29.4,29.5 .31 .3 .31 .9 .45 .6,64.9,68.1,69.9$. 107.1, 114.8. 119.3, 125.9, 126.3. 126.9, 127.1, 128.0. 128.1, 129.1. 129.3, 133.1, 133.6. 135.1, 136.0, 140.7 . 156.8, 158.8. 174.7; $[\alpha]_{\mathrm{D}}^{28}=-12.4(\mathrm{C} 0.13)$ : Anal. Calc. for $\mathrm{C}_{38} \mathrm{H}_{46} \mathrm{O}_{4}: \mathrm{C} 80.88, \mathrm{H} 8.61$ : found $\mathrm{C} 81.20, \mathrm{H} 8.71$.

10h: ${ }^{1} \mathrm{H}-\mathrm{NMR} \delta 0.78-1.87$ (m. $27 \mathrm{H}$ ). 3.84 (q. $\mathrm{IH} . J=7.0$ $\mathrm{Hz}) .4 .02-4.09(\mathrm{~m}, 4 \mathrm{H}), 5.20$ (s. $2 \mathrm{H}) .6 .97-7.75(\mathrm{~m}, 13 \mathrm{H})$ : ${ }^{19} \mathrm{~F}-\mathrm{NMR} \quad 134.63$ : MS: $\mathrm{m} / \mathrm{z} \quad 598.3466 \quad\left[\mathrm{M}^{-} \quad(100 \%)\right.$,

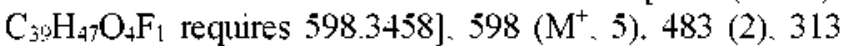
(100). $201(100), 200(24) .71(5 \%):[\alpha]_{0}^{20}=+5.3(\mathrm{C} 0.19)$.

10i. 'H-NMR $\delta 0.78-1.91(\mathrm{~m} .29 \mathrm{H}) .3 .84(\mathrm{q} .1 \mathrm{H} . J=7.0$ Hz). 4.02-4.09 (m. 4H). 5.20 (s. 2H). 6.97-7.75 (m. 13H): ${ }^{19} \mathrm{~F}-\mathrm{NMR}$ 134.61: MS: $\mathrm{m} / \mathrm{z} \quad 612.3610 \quad\left[\mathrm{M}^{-} \quad(100 \%)\right.$. $\mathrm{C}_{410} \mathrm{H}_{49} \mathrm{O}_{4} \mathrm{~F}_{1}$ requires 612.3615], $612\left(\mathrm{M}^{+}, 8\right), 497$ (2), 327
(100), $201(67), 200(14), 71(3 \%):[\alpha]_{[\circ}^{30}=+18.2(\mathrm{C} 0.18)$.

10j: ${ }^{1} \mathrm{H}-\mathrm{NMR} \delta 0.78-1.91(\mathrm{~m}, 3 \mathrm{lH}) .3 .84(\mathrm{q}, \mathrm{lH} . J=7.0$ Hz). 4.02-4.09 (m. 4H), 5.20 (s, 2H). 6.97-7.75 (m. 13H); ${ }^{19} \mathrm{~F}-\mathrm{NMR}$ 134.61: MS: $\mathrm{m} / \mathrm{z} \quad 622.3778 \quad\left[\mathrm{M}^{+}(100 \%)\right.$, $\mathrm{C}_{41} \mathrm{H}_{51} \mathrm{O}_{4} \mathrm{~F}_{1}$ requires $\left.627.377 \mathrm{l}\right] .626\left(\mathrm{M}^{-}, 3\right) .511$ (1). 341 (100), $201(56), 200(12), 71(1 \%):[\alpha]_{0 j}^{2:}=+13.8(\mathrm{C} 0.20)$.

10k: ${ }^{1} \mathrm{H}-\mathrm{NMR} \delta 0.82-1.87(\mathrm{~m}, 29 \mathrm{H}), 3.84$ (q. IH. $J=7.0$ Hz). 4.03-4.12 (m. 4H), 5.19 (s, 2H). 6.97-7.75 (m. 13H); ${ }^{19} \mathrm{~F}-\mathrm{NMR}$ 134.67: MS: $\mathrm{m} / \mathrm{z} 612.3619\left[\mathrm{M}^{-}(\mathrm{I} 00 \%), \mathrm{C}_{41 \mathrm{i}} \mathrm{H}_{4} \mathrm{O}_{4} \mathrm{~F}_{1}\right.$ requires 612.3615$] .612\left(\mathrm{M}^{-}, 3\right) .511$ (1). $341(100), 201$ (69). $200(14) .57(13 \%):[\alpha]_{[j}^{2 ?}=+17.3(\mathrm{C} 0.21)$.

10l: ${ }^{1} \mathrm{H}-\mathrm{NMR} \delta 0.78-1.87(\mathrm{~m}, 33 \mathrm{H}) .3 .84$ (q. $1 \mathrm{H}, J=7.0$ Hz). 4.03-4.09 (m. 4H), 5.20 (s, 2H). 6.97-7.75 (m. 13H); ${ }^{19} \mathrm{~F}-\mathrm{NMR}$ 134.63: MS: $\mathrm{m} / \mathrm{z} \quad 640.3926 \quad\left[\mathrm{M}^{+} \quad(100 \%)\right.$, $\mathrm{C}_{42} \mathrm{H}_{33} \mathrm{O}_{4} \mathrm{~F}_{1}$ requires 640.3928$] .640\left(\mathrm{M}^{-}, 12\right) .5 \mathrm{ll}(2), 341$ $(100), 201(63), 200(13 \%) ;[\alpha]_{[j}^{2 ?}=+12.7(\mathrm{C} 0.27)$.

Acknowledgements. This work was performed as part of Advanced Backbone IT Technology Development Project supported by Ministry of Information \& Conmunication in Republic of Korea.

\section{References}

1. Goodby, J. W: Slaney, A. J.: Booth, C. J: Nishivama, I.: Vuijk. J D.; Styring. P.: Toyne, K. J. Mol. Crust. Liq. Crust. 1994, 243, 231.

2. (a) Ema. K.: Kanai. M.: Yao. H.: Takanishi. Y.: Takezoe. H. Phus. Rev E 2000. 61. 1585. (b) Guillon. D.: Cherkaoui. M. Z: Sebastiao. P.: Mery. S.: Nicoud. T. F.: Galerne. Y. ACS Smposim Series 2002. 798 (Anisotropic Organic Materials). 214.

3. (a) A Practical Approach to Chiral Separation by Liquid Chromatographys Subramanian, G.. Ed: VCH: Weinheim. 1994 (b) Hyun. M.: Minl. H. J.: Cho. Y. J. Bull. Korean Chem. Soc. 2003. 24. 911 . (c) Kim. I. W.: Ryu. I. K.: Ahn. S. D.: Park. J. H.: Lee. K.-P.: Ryoo. I. I.: Hyun. M.: Okamoto. Y: Yamamoto. C. Carr. P. W. Bull Korean Chem. Soc. 2003. 24. 239. (d) Hyun. M:Cho. Y. J.: Baik, I.-K. Bull. Korem Chem. Soc. 2002. 23. 1291.

4. Kang. K.-T.: Kim. C. H.: Lee. S. K.: Shin. M. S.: Lee. K. U.: Lee. J. G.: Choi. J. W.: Kim. Y. B. Ferroelectrics 2002. 276. 55.

5. Hyun. M.: Jit1. J. S.: Ryoo. I. J. Bull. Korean Chem. Soc. 1998. 19. 1392.

6. Ebbers. E. J.: Ariaans, G. J. A; Bruggink. A.: Zwanenburg, B Tetrahedronitsymetoy 1999, 10. 3701 .

7. Wul. S.-L.: Hsieh. W.-J. Chem Hater 1999. 11.852

8. Wu. S.-L.:Yen. P.-C.: Hsieh. W.-I. Liq. Const 1998. 24. 741 .

9. Hsieh. W.-J.: Wu. S.L. Mol. Cost Liq. Crnst 1997. 302.253.

10. Wu. S.-L.; Hsieh. W.-J. Liq. Crist 1996. 21,783.

11. Wu. S.-L.; Chiang. C. T. Liq. Chrt 2002, $29,39$.

12. Pirkle. W. H.: Welch, C. J. J. Liq. Chomatogr. 1991. 9. 413 